Agro-Science Journal of Tropical Agriculture, Food, Environment and Extension Volume 20 Number 1 (January 2021) pp. 1 - 8

ISSN 1119-7455

\title{
EFFICACY OF THREE BOTANICALS ON POSTHARVEST FUNGAL CONTAMINANTS OF MELON (Citrullus colocynthis) KERNELS
}

\author{
${ }^{* 1,2}$ Obani F.T. and ${ }^{1}$ Ikotun B. \\ ${ }^{1}$ Department of Crop Protection \& Environmental Biology, University of Ibadan, Ibadan, Nigeria \\ ${ }^{2}$ Michael Okpara University of Agriculture Umudike, Abia State, Nigeria \\ *Corresponding author's email: tochyfloxy@gmail.com
}

\begin{abstract}
Melon (Citrullus colocynthis L.) is an important crop used mainly for soups in Nigeria. Egusi: as it is commonly called in Nigeria is contaminated by many fungal pathogens which reduce quality of seeds during storage. Use of botanicals can be a safe method to manage fungal contamination instead of chemicals which pose threat to human health. Therefore, efficacy of Piper guineense, Xylopia aethiopica and Ocimum gratissimum on fungi in shelled Egusi seed kernels (EK) were evaluated. One market in each of six Southwestern Nigerian states where Egusi is sold was purposively selected in 2012 and 2013. Egusi kernels $(1 / 2 \mathrm{~kg}, n=162)$ were purchased from selected traders for fungi isolation, identification and incidence (\%) determination. Clean EK treated with botanical powder $\left(10,20\right.$ and $\left.40 \mathrm{~g} \mathrm{~kg}^{-1}\right)$ were inoculated with Aspergillus flavus, A. niger, A. tamarii, Rhizopus sp., Penicillium aurantiogriseum, P. citrinum and Fusarium solani biweekly for 14-week storage period to evaluate growth reduction (\%). Control was inoculated with sterile distilled water. Aspergillus flavus, A. niger, A. tamarii, Penicillium citrinum, P. aurantiogriseum, Fusarium solani and Rhizopus sp. were frequently encountered in EK. Aspergillus $(32.4 \pm 1.6 \%)$ was the most predominant fungus followed by Rhizopus (21.5 $\pm 2.0 \%)$ in all States. Piper guineense $\left(40 \mathrm{~g} \mathrm{~kg}^{-1}\right)$, X. aethiopica $\left(20 \mathrm{~g} \mathrm{~kg}^{-1}\right)$ and $O$. gratissimum $\left(10 \mathrm{~g} \mathrm{~kg}^{-1}\right)$ powders significantly reduced aflatoxin contamination by $42.5 \%, 56.5 \%$ and $45.0 \%$, respectively; fungi growths were progressively reduced by $P$. guineense (5.5-90.0\%), X. aethiopica (6.7-100.0\%) and 0 . gratissimum (7.4-66.7\%) up to 12 weeks of storage. Egusi kernels were highly infected with postharvest fungi especially Aspergillus species. Xylopia aethiopica at $20 \mathrm{~g} \mathrm{~kg}^{-1}$, O. gratissimum (40.0\%) and P. guineense (40.0\%) reduced fungi growth on Egusi considerably and therefore could be used as a safe management option to mitigate storage fungi contamination in Egusi kernels.
\end{abstract}

Key words: storage fungi, botanical powder, Southwest Nigeria, fungi contamination

\section{INTRODUCTION}

Melon (Citrullus colocynthis L.) commonly known as Egusi is an important soup spice produced in abundance in different parts of Nigeria and many countries in sub-Saharan Africa (Van der Vossen et al., 2004). The oil rich seeds are the economic part of the crop. It is also rich in protein and contains good quantities of most of the essential amino acids (Ayodele and Salami, 2006).

The seeds can be used or processed by a variety of forms such as soaking, boiling, fermentation, grinding and molding into balls before use for soup preparation. Egusi is not only valuable as a staple for local consumption, but also as export commodity to be sold to people who have emigrated from Africa to other continents of the world (Van der Vossen et al., 2004).

Many groups of fungi are known to contaminate Egusi seeds during storage. They reduce seed storability, quality, export and marketability potentials; and above all deposit a large number of metabolites in the seeds; some of which are toxic to humans (Chiejina, 2006; Atehnkeng et al., 2008). Fungi species belonging to the genera Rhizopus, Penicillium amongst others have been reported as seed pathogens of Nigerian stored Egusi seed. Many storage fungi that have been variously implicated in the spoilage of fruits and vegetables have been isolated from Egusi seeds by various authors (Chiejina, 2006; Aboloma et al., 2009; Aboloma et al., 2012). Fungal deterioration of seeds occurs in form of rot, sclerotization of seed, and seed discolourization (Shetty 1992).

Prior to harvest of Egusi seeds, fungal infection may not be up to the level that can lead to economic damage. Small quantities of spores of storage fungi may be present on grain meant for storage or may be present on spilled grains present in storage equipment or structures. This small amount of inoculum can multiply rapidly leading to significant grain infection under poor storage conditions. Bankole (1993) reported as many as 13 storage fungal species in stored Egusi seeds. Also, Chiejina (2006) isolated thirteen different fungal species and 
two unidentified species from Egusi seed kernel. These fungi are widely distributed and almost always present on the seeds during storage. However, the development of these fungi are influenced by the moisture content, the temperature, the condition of the Egusi seed going into storage, the length of time the seed is stored and the amount of insect and mite activity in the stored seed.

Interestingly, nature supplies a reasonable number of plant products that have useful properties for crop protection, which are often neglected in favour of commercial products. These natural substances cause little disturbance to the natural balance between living organisms. They are cheap and can be produced by farmers from local sources. They are often harmless to humans and animals and are rarely toxic to plants when compared with artificial (Dusanee, 2011).

The use of benzoic acid, gamma-irradiation and fumigants to prevent postharvest fungal contamination have been emphasized and in use but they are not safe for crops meant for human consumption. Treatment with natural products from plants or herbs (botanicals) which are edible prior to storage is a safe option for grains meant for humans. The botanicals are mostly available locally and are not potential environmental and biological hazards. The possibilities of using botanical pesticides seem almost endless and their potentials can be fully exploited (Yallappa et al., 2012). The prevention of postharvest fungal contamination in Egusi is one of the best and most effective strategies to reduce yield loss and maximize income from harvested seeds; hence the need to assess the potentials of some medicinal plants for the control of storage fungi in stored Egusi seed kernels. The objective of this study therefore was to determine the effects of selected botanicals on fungal growth in treated Egusi seed kernels.

\section{MATERIALS AND METHODS Sample Collection}

A total of 162 melon vendors were randomly selected in each of the six South-western Nigerian States (Sabo in Ondo, Sabo in Ekiti, Oto in Lagos, Sabo in Ogun, Oja Oba in Osun, Bodija in Oyo) in 2012 and 2013. Shelled melon seeds (kernel) were purchased directly from a major market where melon from the producing areas is unloaded in each of the six south-western states of Nigeria. For each market visited Simple random sampling was adopted for sample collection. From each trader, $0.5-1 \mathrm{~kg}$ of melon seed was purchased and taken to the laboratory for studies. Various melon seeds were purchased from three traders at different points within the same market, packed into different polythene bags and these served as the replicates. For each bag, sampled melon kernels were collected at different points in the bag to form a composite sample.

\section{Preparation of Botanical Powder}

The botanicals were purchased from the Ojoo market. The botanicals were thoroughly washed, airdried under shade until they were properly dried; ground to fine powder using the Warring laboratory blender (Warring Commercial, Springfield, MO) and stored at $4^{\circ} \mathrm{C}$ until when needed.

\section{Application of Plant Powders to Egusi Kernels and Fungal Contamination Test}

The botanical powders were prepared as already described above; the botanical powders were used to dust $1 \mathrm{~kg}$ of clean (uninfected) Egusi kernels in plastic woven bags, mixed properly and placed on the shelve for a period of three and half months. Prior to treatment and storage, shelled melon kernels were dried to moisture content of $10 \%$ (determined with Pfeuffer helite moisture meter). The controls were not dusted with any botanical powder (Bankole and Joda, 2004). Subsamples of 50 $\mathrm{g}$ were collected from each $1 \mathrm{~kg}$ sample at $2 \mathrm{nd}, 4 \mathrm{th}$, 6 th, 8th 10th 12th and 14th week for fungal contamination test with $A$. flavus and other identified predominant fungal species ( $A$. niger, $A$. tamarii, Rhizopus sp., $P$. aurantiogriseum, $P$. citrinum and $F$. solani). Inocula suspensions were prepared from fresh, mature (5-day-old) fungi cultures. Fungal colonies were covered with $5 \mathrm{ml}$ of distilled sterile water containing 1\% Tween 20 per $100 \mathrm{ml}$ to enhance uniform spore dispersal for hydrophobic genus such as Apergillus. The final inoculum size was adjusted to a concentration of $1.0 \times 10^{6} \mathrm{spore} / \mathrm{ml}$ by microscopic enumeration with a cell-counting haemocytometer (Aberkane et al., 2002). The Egusi kernels treated with botanicals were washed in three changes of sterile distilled water and then twenty kernels were inoculated with $100 \mu \mathrm{L}$ of spore suspension of each test fungi. Five kernels were plated on petri dishes containing solidified Potato Dextrose Agar (PDA) and incubated at room temperature for five days. The controls were plated on PDA without inoculation with any fungal species. Percentage kernel colonization was recorded after incubation.

\section{Isolation and Identification of Fungi from Market Melon Samples}

Egusi seed kernels from traders' shop were processed and fungi isolated done following the methods described by (Atehnkeng et al., 2014) using PDA in which $0.05 \mathrm{ml}$ of lactic acid had been added to suppress bacterial growth (Atehnkeng et al., 2008). After incubation for 5 days at room temperature, the colony forming units (cfu $\mathrm{ml}^{-1}$ ) of each fungal species identified was determined by counting the number of colonies formed. Axenic culture of each isolate was obtained by sub culturing on fresh PDA plates. Identification of the isolated fungi was done based on colony morphology and microscopic examination which 
were compared with the literature. Slides were prepared from fungal colonies produced on the medium for identification of the organisms using mycological reference books and research articles (Barnett and Hunter, 1999; Alexopoulos et al., 2002; Samson et al., 2004) and the descriptions of Barnett and Hunter (1999). The experiments were carried out with treatment in triplicates laid out in completely randomized design.

\section{Determination of Percentage Occurrence of the Fungal Isolates}

This was done to determine the incidence of occurrence of the different fungal isolates. The total number of each isolate in all samples was obtained against the total number of all the isolates in all the samples screened. Frequency of occurrence was determined using the method described by Giridher and Ready (1997):

Percentage of frequency $=$

No. of observations in which a species appeared $\times 100$

$$
\text { Total no. of observations }
$$

\section{Data Analysis}

Data on fungal incidence in melon grains were analyzed using SAS (version 9.2, SAS Institute Inc., Cary, NC). The means were separated using Fisher's protected least significant difference (LSD) test to determine significant differences among the means obtained from the different states or treatments.

\section{RESULTS}

Nine different fungal genera apart from Aspergillus were identified in the melon samples collected in 2012. They are Fusarium, Penicillium, Rhizopus, Botryodiplodia, Trichoderma, Alternaria, Sclerotium, Cladosporium and Macrophomina. Across the six states, Aspergillus species were the most predominant fungal species identified, followed by species belonging to the genera Rhizopus, Fusarium, Alternaria, and Penicillium while Trichoderma,
Botryodiplodia and Macrophomina species were the least predominant. Aspergillus species had the highest fungal colonies per gram ( $\mathrm{cfu} \mathrm{g}^{-1}$ ) and was significantly $(p=0.05)$ higher than all other fungal genera identified. The highest fungal colonies per gram (cfu g $\mathrm{g}^{-1}$ ) of Aspergillus species was recorded in samples from Osun State $\left(11,920 \mathrm{cfu}^{-1}\right)$ while the least cfu $\mathrm{g}^{-1}$ was recorded in Lagos State and they were significantly different across the states. The highest cfu $\mathrm{g}^{-1}$ of Fusarium, Botryodiplodia, Alternaria, Sclerotium and Cladosporium were isolated from samples from Lagos State. The highest cfu $\mathrm{g}^{-1}$ of Penicillium and Rhizopus were found in Ondo (2193.3 cfu g ${ }^{-1}$ ) and Ogun (766.7 $\mathrm{cfu} \mathrm{g}^{-1}$ ) States, respectively (Table 1).

Aspergillus, Fusarium, Penicillium, Rhizopus, Botryodiplodia, Paecilomyces, Alternaria, Sclerotium and Macrophomina were identified in the melon samples collected during 2013 sampling (Table 1). Aspergillus species were the most predominant fungal species identified across the six states, followed by species belonging to the genera Rhizopus, Penicillium, Fusarium, Alternaria, Sclerotium, while Cladosporium, Botryodiplodia and Macrophomina species were the least predominant. Aspergillus species had the highest fungal colonies per gram ( $\mathrm{cfu}^{-1}$ ) and was significantly $(p=0.05)$ higher than all other fungal genera identified except in Ondo where the highest Rhizopus colonies was recorded (11990.7 $\mathrm{cfu} \mathrm{g}^{-1}$ ), and also in Ogun State (2466.7 $\left.\mathrm{cfu} \mathrm{g}^{-1}\right)$. The highest fungal colonies per gram (cfu g-1) of Aspergillus species was recorded in samples from Oyo State (3250 $\mathrm{cfu} \mathrm{g}^{-1}$ ) while the least cfu $\mathrm{g}^{-1}$ was recorded in Ekiti State and they were significantly different across the states. The highest $\mathrm{cfu} \mathrm{g}^{-1}$ of Penicillium, Fusarium, Botryodiplodia, Alternaria, and Paecilomysis were isolated from samples collected from Ogun State. The highest cfu g $\mathrm{g}^{-1}$ of Sclerotium was recorded in Osun while the highest $\mathrm{cfu} \mathrm{g}^{-1}$ of Cladosporium occurred in Lagos $\left(22.2 \mathrm{cfu} \mathrm{g}^{-1}\right)$ and Ogun (22.2 $\left.\mathrm{cfu} \mathrm{g}^{-1}\right)$ States (Table 1).

Table 1: Mould content of Egusi kernels collected from the six states of South-western Nigeria in 2012 and 2013

\begin{tabular}{|c|c|c|c|c|c|c|c|c|c|c|c|}
\hline \multicolumn{12}{|c|}{ Fungal colonies $\left(\mathrm{cfu} \mathrm{g}^{-1}\right)$} \\
\hline Year & State & Asp & Rhz & Pen & Bot & Fus & Cld & Alt & Scl & Pec & Tri \\
\hline \multirow{6}{*}{2012} & Ekiti & $2406.7^{b}$ & $444.4 a^{b}$ & $155.6^{\mathrm{c}}$ & $22.2 b^{c}$ & $22.2^{\mathrm{c}}$ & $22.2^{\mathrm{c}}$ & $22.2^{\mathrm{c}}$ & $22.2^{\mathrm{c}}$ & $0.0^{\mathrm{a}}$ & $0.0^{\mathrm{b}}$ \\
\hline & Lagos & $2366.7^{b}$ & $188.9^{\mathrm{c}}$ & $355.6^{\mathrm{c}}$ & $500.0^{\mathrm{a}}$ & $500.0^{\mathrm{a}}$ & $500.0^{\mathrm{a}}$ & $500.0^{\mathrm{a}}$ & $500.0^{\mathrm{a}}$ & $0.0^{\mathrm{a}}$ & $100.0^{\mathrm{a}}$ \\
\hline & Ogun & $3128.3^{b}$ & $766.7^{\mathrm{a}}$ & $513.3^{\mathrm{c}}$ & $0.0^{\mathrm{c}}$ & $20.0^{\mathrm{c}}$ & $0.0^{\mathrm{c}}$ & $0.0^{\mathrm{c}}$ & $0.0^{\mathrm{c}}$ & $0.0^{\mathrm{a}}$ & $100.0^{\mathrm{a}}$ \\
\hline & Ondo & $4600.0^{b}$ & $122.2^{\mathrm{c}}$ & $2193.3^{\mathrm{a}}$ & $120.0 \mathrm{a}^{\mathrm{b}}$ & $120.0^{\mathrm{c}}$ & $500.0^{\mathrm{a}}$ & $120.0^{\mathrm{b}}$ & $120.0^{\mathrm{b}}$ & $0.0^{\mathrm{a}}$ & $0.0^{\mathrm{b}}$ \\
\hline & Osun & $11920.0^{\mathrm{a}}$ & $322.2^{\mathrm{b}}$ & $1753.3 \mathrm{~b}^{\mathrm{a}}$ & $158.0 \mathrm{a}^{\mathrm{b}}$ & $126.7 \mathrm{ab}^{\mathrm{c}}$ & $120.0^{\mathrm{b}}$ & $126.7^{\mathrm{b}}$ & $126.7^{\mathrm{b}}$ & $0.0^{\mathrm{a}}$ & $100.0^{\mathrm{a}}$ \\
\hline & Oyo & $5794.7^{b}$ & $133.3^{\mathrm{c}}$ & $1005.6 \mathrm{a}^{\mathrm{b}}$ & $94.4 \mathrm{a}^{\mathrm{b}}$ & $188.9^{\mathrm{ab}}$ & $88.9^{\mathrm{b}}$ & $89.0^{\mathrm{b}}$ & $88.9^{\mathrm{b}}$ & $0.0^{\mathrm{a}}$ & $0.0^{\mathrm{b}}$ \\
\hline \multirow{6}{*}{2013} & Ekiti & $1511.1^{b}$ & $383.3^{\mathrm{c}}$ & $372.2 b$ & $0.0^{\mathrm{b}}$ & $5.6^{\mathrm{c}}$ & $0.0^{\mathrm{b}}$ & $55.6^{\mathrm{b}}$ & $0.0^{\mathrm{c}}$ & $0.0^{\mathrm{b}}$ & $0.0^{\mathrm{a}}$ \\
\hline & Lagos & $2116.7^{b}$ & $225.0^{\mathrm{c}}$ & $8.3^{\mathrm{c}}$ & $0.0^{\mathrm{b}}$ & $5.6^{\mathrm{c}}$ & $22.2^{\mathrm{a}}$ & $25.0^{\mathrm{b}}$ & $0.0^{\mathrm{c}}$ & $0.0^{\mathrm{b}}$ & $0.0^{\mathrm{a}}$ \\
\hline & Ogun & $2230.3^{\mathrm{ab}}$ & $2466.7^{\mathrm{ab}}$ & $2076.3^{a}$ & $100.0^{\mathrm{a}}$ & $1868.7^{\mathrm{a}}$ & $6.7^{\mathrm{b}}$ & $880.0^{\mathrm{a}}$ & $126.7^{\mathrm{a}}$ & $266.7^{\mathrm{a}}$ & $0.0^{\mathrm{a}}$ \\
\hline & Ondo & $2736.7^{\mathrm{ab}}$ & $11990.7 \mathrm{a}$ & $1923.6^{\mathrm{ab}}$ & $100.0^{\mathrm{a}}$ & $873.6^{\mathrm{b}}$ & $22.2^{\mathrm{a}}$ & $116.7^{\mathrm{ab}}$ & $50.0^{\mathrm{b}}$ & $6.7^{\mathrm{b}}$ & $0.0^{\mathrm{a}}$ \\
\hline & Osun & $2380.0^{\mathrm{ab}}$ & $1055.6^{\mathrm{b}}$ & $350.0^{\mathrm{b}}$ & $0.0^{\mathrm{b}}$ & $50.0^{\mathrm{c}}$ & $8.3^{\mathrm{b}}$ & $0.0^{\mathrm{b}}$ & $166.7^{\mathrm{a}}$ & $0.0^{\mathrm{b}}$ & $0.0^{\mathrm{a}}$ \\
\hline & Oyo & $3250.0^{\mathrm{a}}$ & $2622.2^{\mathrm{ab}}$ & $566.7^{\mathrm{b}}$ & $0.0^{\mathrm{b}}$ & $50.0^{\mathrm{c}}$ & $0.0^{\mathrm{b}}$ & $0.0^{\mathrm{b}}$ & $150.0^{\mathrm{a}}$ & $111.1^{\mathrm{ab}}$ & $0.0^{\mathrm{a}}$ \\
\hline
\end{tabular}




\section{Incidence of Fungal Species in Egusi Kernels} Aspergillus species had the highest percentage incidence across all the states, followed by Penicillium and Fusarium species while Macrophomina was the least in 2012 (Figure 1). In Ekiti and Ogun States, significant differences were not observed in the incidence of Fusarium and Alternaria species. The same trend was observed in Fusarium and Penicillium species in Lagos and Osun States. In 2013, Aspergillus species also had the highest percentage incidence across all the states, followed by Rhizopus and Penicillium species while Paecilomyces was the least. The incidence of Aspergillus species was significantly $(p=0.05)$ higher than all other fungal genera identified except in Osun State where Aspergillus and Rhizopus species were significantly $(p=0.05)$ higher than all other fungal genera identified in both 2012 and 2013 (Figures 1 and 2).

\section{Effect of Selected Botanicals on Growth of} Fungal Contaminants in Treated Egusi kernels Storing Egusi kernels together with botanical powders significantly reduced fungal growth in the stored Egusi kernels. All the botanicals tested differed from each other in their ability to reduce fungal growth on the seeds. The result presented in Table 2 shows the percentage reduction of fungal growth in Egusi seeds treated with different concentrations of $P$. guineense. Forty-gram $\left(40 \mathrm{~g} \mathrm{~kg}^{-1}\right)$ treatment generally had the highest growth reduction for the various fungal isolates except for $F$. solani where 10 and $20 \mathrm{~g} \mathrm{~kg}^{-1}$ treatment gave the highest growth reduction of $60.0 \%$ while $40 \mathrm{~g} \mathrm{~kg}^{-1}$ had the least growth reduction $(10.0 \%)$. Percentage growth reduction declined gradually as the storage period increased. One month after treatment, the botanicals were not effective in inhibiting the growth of Rhizopus sp. The growth of $P$. aurantiogriseum was inhibited effectively up to $8^{\text {th }}$ week, $10^{\text {th }}$ week and $12^{\text {th }}$ week after storage at 10 , 20 and $40 \mathrm{~g} \mathrm{~kg}^{-1}$ treatment, respectively. P. citrinum growth was effectively reduced by more than $20.0 \%$ by the various levels of the botanical treatments throughout the 14 weeks of Egusi storage. The second experiment gave similar results. Treatment at $40 \mathrm{~g} \mathrm{~kg}^{-1}$ generally had the highest growth reduction for the various fungal isolates except for $F$. solani where 10 and $20 \mathrm{~g} \mathrm{~kg}^{-1}$ treatment gave the higher growth reductions of $55.0 \%$ and $65.0 \%$, respectively than $40 \mathrm{~g} \mathrm{~kg}^{-1}$ which had the least growth reduction of $15.0 \%$. P. citrinum growth was still effectively reduced by more than $20.0 \%$ by the various levels of the botanical treatments throughout the period of 14 weeks of Egusi storage (Table 2).

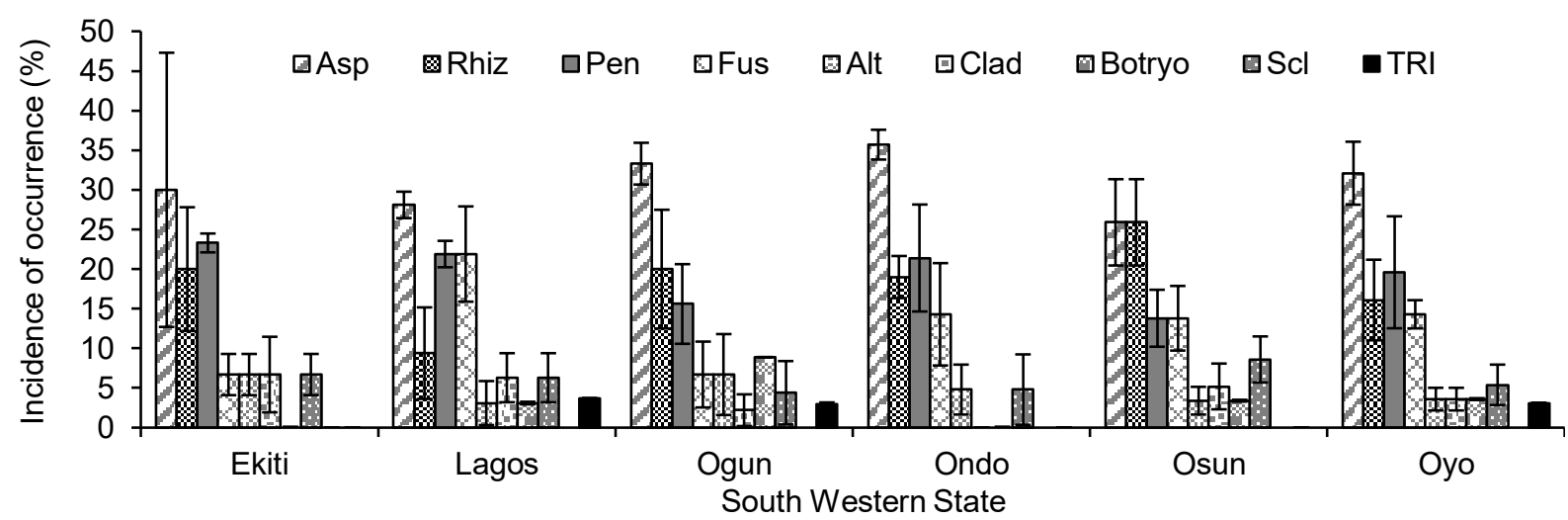

Figure 1: Percentage incidence of fungal species isolated from Egusi kernels collected from six states in South-western Nigeria in 2012. Asp - Aspergillus; Pen - Penicillium; Fus - Fusarium; Rhiz - Rhozopus; Botryo - Botryodiplodia; Tri - Trichoderma; Clad-Cladosporium; Alt - Alternaria; Scl - Sclerotium. For each bar, the vertical line represents the standard error of the means.

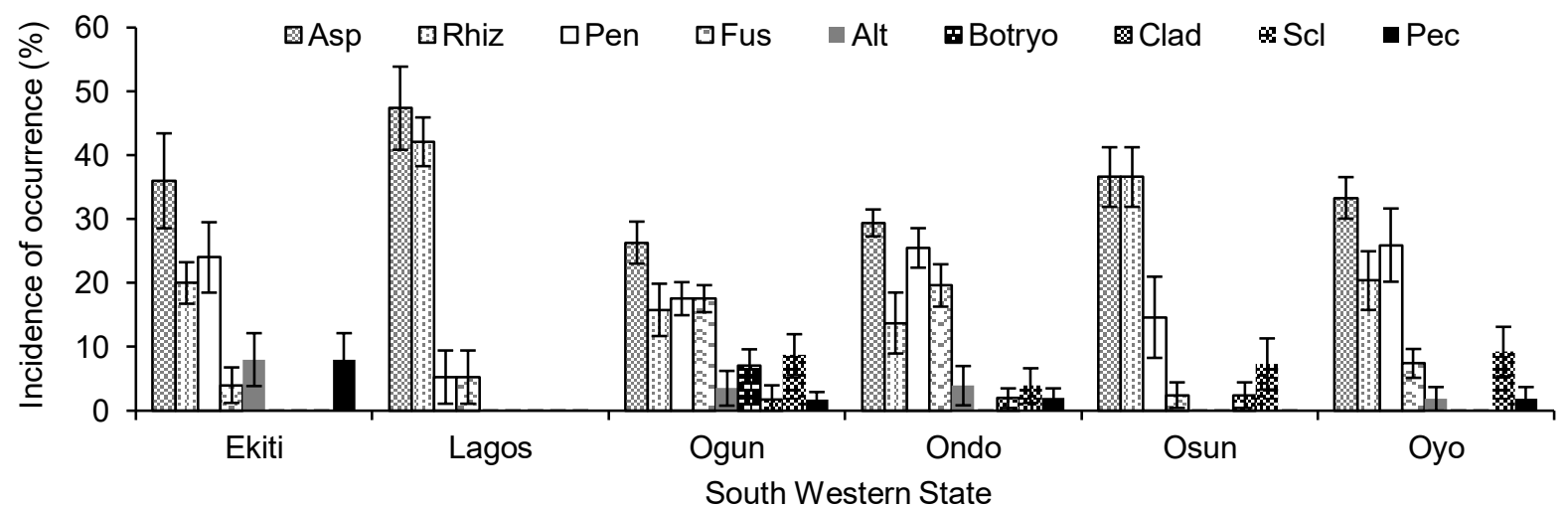

Figure 2: Percentage incidence of fungal species isolated from Egusi kernels collected from six states in South-western Nigeria in 2013. Asp - Aspergillus; Pen - Penicillium; Fus - Fusarium; Rhiz - Rhozopus; Botryo - Botryodiplodia; Tri - Trichoderma;

Clad - Cladosporium; Alt - Alternaria; Scl-Sclerotium; Pec-Paecilomysis. For each bar, the vertical line represents the standard error of the means. 
Table 2: Effect of different concentrations of $P$. guineense on fungal contaminants of stored Egusi kernels at different weeks after storage

\begin{tabular}{|c|c|c|c|c|c|c|c|c|c|c|c|c|c|c|c|}
\hline \multirow{3}{*}{$\begin{array}{l}\frac{\tilde{u}}{d} \\
\frac{d}{3}\end{array}$} & $\begin{array}{l}\widetilde{b_{00}} \\
\frac{0}{000}\end{array}$ & \multicolumn{14}{|c|}{ Percentage growth reduction } \\
\hline & & \multicolumn{5}{|c|}{ First experiment (2012) } & \multirow[b]{2}{*}{ TAM } & \multirow[b]{2}{*}{ YEL } & \multirow[b]{2}{*}{ FLV } & \multirow[b]{2}{*}{ NIG } & \multicolumn{3}{|c|}{ Second experiment (2013) } & \multirow[b]{2}{*}{ TAM } & \multirow[b]{2}{*}{ YEL } \\
\hline & & FLV & NIG & PUR & RHZ & SOL & & & & & PUR & RHZ & SOL & & \\
\hline \multirow[t]{3}{*}{2} & 10 & $25.0 \mathrm{~b}$ & $20.0 \mathrm{a}$ & $70.0 \mathrm{~b}$ & $20.0 \mathrm{c}$ & $60.0 \mathrm{a}$ & $25.0 \mathrm{~b}$ & $90.0 \mathrm{a}$ & $25.0 \mathrm{~b}$ & $25.0 \mathrm{a}$ & $85.0 \mathrm{~b}$ & $20.0 \mathrm{~b}$ & $55.0 \mathrm{a}$ & $40.0 \mathrm{a}$ & $90.0 \mathrm{~b}$ \\
\hline & 20 & $55.0 \mathrm{a}$ & $50.0 \mathrm{a}$ & $100.0 \mathrm{a}$ & $30.0 \mathrm{~b}$ & $60.0 \mathrm{a}$ & $50.0 \mathrm{a}$ & $100.0 \mathrm{a}$ & $60.0 \mathrm{a}$ & $40.0 \mathrm{a}$ & $100.0 \mathrm{a}$ & $30.0 \mathrm{~b}$ & $65.0 \mathrm{a}$ & $40.0 \mathrm{a}$ & $100.0 \mathrm{a}$ \\
\hline & 40 & $50.0 \mathrm{a}$ & $40.0 \mathrm{a}$ & $90.0 \mathrm{a}$ & $60.0 \mathrm{a}$ & $10.0 \mathrm{~b}$ & $45.0 \mathrm{a}$ & $100.0 \mathrm{a}$ & $60.0 \mathrm{a}$ & $35.0 \mathrm{a}$ & $95.0 \mathrm{ab}$ & $60.0 \mathrm{a}$ & $15.0 \mathrm{~b}$ & $35.0 \mathrm{a}$ & $100.0 \mathrm{a}$ \\
\hline \multirow[t]{3}{*}{4} & 10 & $25.0 \mathrm{~b}$ & $35.0 \mathrm{~b}$ & $75.0 \mathrm{a}$ & $0.0 \mathrm{a}$ & $15.0 \mathrm{a}$ & $50.0 \mathrm{a}$ & $95.0 \mathrm{c}$ & $20.0 \mathrm{a}$ & $30.0 \mathrm{~b}$ & $88.9 \mathrm{a}$ & $0.0 \mathrm{a}$ & $10.0 \mathrm{a}$ & $40.0 \mathrm{a}$ & $80.0 \mathrm{~b}$ \\
\hline & 20 & $30.0 \mathrm{a}$ & $25.0 \mathrm{~b}$ & $95.0 \mathrm{a}$ & $0.0 \mathrm{a}$ & $10.0 \mathrm{a}$ & $35.0 \mathrm{a}$ & $95.0 \mathrm{~b}$ & $40.0 \mathrm{a}$ & $35.0 \mathrm{ab}$ & $95.0 \mathrm{a}$ & $0.0 \mathrm{a}$ & $25.0 \mathrm{a}$ & $25.0 \mathrm{a}$ & $80.0 \mathrm{~b}$ \\
\hline & 40 & $50.0 \mathrm{a}$ & $65.0 \mathrm{a}$ & $100.0 \mathrm{a}$ & $5.0 \mathrm{a}$ & $25.0 \mathrm{a}$ & $45.0 \mathrm{a}$ & $100.0 \mathrm{a}$ & $40.0 \mathrm{a}$ & $65.0 \mathrm{a}$ & $100.0 \mathrm{a}$ & $5.0 \mathrm{a}$ & $40.0 \mathrm{a}$ & $45.0 \mathrm{a}$ & $100.0 \mathrm{a}$ \\
\hline \multirow[t]{3}{*}{6} & 10 & $0.0 \mathrm{a}$ & $15.0 \mathrm{a}$ & $40.0 \mathrm{a}$ & $0.0 \mathrm{a}$ & $10.0 \mathrm{a}$ & $10.0 \mathrm{a}$ & $80.0 \mathrm{~b}$ & $5.0 \mathrm{a}$ & $30.0 \mathrm{a}$ & $35.0 \mathrm{~b}$ & $0.0 \mathrm{a}$ & $5.0 \mathrm{a}$ & $15.0 \mathrm{a}$ & $80.0 \mathrm{~b}$ \\
\hline & 20 & $20.0 \mathrm{a}$ & $35.0 \mathrm{a}$ & $58.3 \mathrm{a}$ & $0.0 \mathrm{a}$ & $5.0 \mathrm{~b}$ & $0.0 \mathrm{~b}$ & $80.0 \mathrm{~b}$ & $10.0 \mathrm{a}$ & $30.0 \mathrm{a}$ & $58.3 \mathrm{a}$ & $0.0 \mathrm{a}$ & $0.0 \mathrm{a}$ & $0.0 \mathrm{a}$ & $80.0 \mathrm{~b}$ \\
\hline & 40 & $10.0 \mathrm{a}$ & $25.0 \mathrm{a}$ & $65.0 \mathrm{a}$ & $0.0 \mathrm{a}$ & $5.0 \mathrm{~b}$ & $5.0 \mathrm{a}$ & $90.0 \mathrm{a}$ & $10.0 \mathrm{a}$ & $40.0 \mathrm{a}$ & $60.0 \mathrm{a}$ & $0.0 \mathrm{a}$ & $5.0 \mathrm{a}$ & $5.0 \mathrm{a}$ & $90.0 \mathrm{a}$ \\
\hline \multirow[t]{3}{*}{8} & 10 & $0.0 \mathrm{~b}$ & $15.0 \mathrm{~b}$ & $35.0 \mathrm{~b}$ & $0.0 \mathrm{a}$ & $5.0 \mathrm{a}$ & $5.0 \mathrm{a}$ & $65.0 \mathrm{~b}$ & $5.0 \mathrm{a}$ & $15.0 \mathrm{a}$ & $30.0 \mathrm{~b}$ & $0.0 \mathrm{a}$ & $10.0 \mathrm{a}$ & $10.0 \mathrm{a}$ & $70.0 \mathrm{~b}$ \\
\hline & 20 & $10.0 \mathrm{ab}$ & $10.0 \mathrm{ab}$ & $45.0 \mathrm{ab}$ & $0.0 \mathrm{a}$ & $0.0 \mathrm{a}$ & $0.0 \mathrm{a}$ & $85.0 \mathrm{ab}$ & $10.0 \mathrm{a}$ & $5.0 \mathrm{a}$ & $40.0 \mathrm{~b}$ & $0.0 \mathrm{a}$ & $0.0 \mathrm{a}$ & $0.0 \mathrm{a}$ & $85.0 \mathrm{ab}$ \\
\hline & 40 & $15.0 \mathrm{a}$ & $25.0 \mathrm{a}$ & $70.0 \mathrm{a}$ & $0.0 \mathrm{a}$ & $5.0 \mathrm{a}$ & $0.0 \mathrm{a}$ & $90.0 \mathrm{a}$ & $15.0 \mathrm{a}$ & $30.0 \mathrm{a}$ & $64.3 \mathrm{a}$ & $0.0 \mathrm{a}$ & $10.0 \mathrm{a}$ & $0.0 \mathrm{a}$ & $90.0 \mathrm{a}$ \\
\hline \multirow[t]{3}{*}{10} & 10 & $0.0 \mathrm{a}$ & $15.0 \mathrm{a}$ & $15.0 \mathrm{c}$ & $0.0 \mathrm{a}$ & $5.0 \mathrm{a}$ & $0.0 \mathrm{a}$ & $65.0 \mathrm{~b}$ & $0.0 \mathrm{a}$ & $5.0 \mathrm{a}$ & $15.0 \mathrm{~b}$ & $0.0 \mathrm{a}$ & $5.0 \mathrm{a}$ & $5.0 \mathrm{a}$ & $70.0 \mathrm{~b}$ \\
\hline & 20 & $0.0 \mathrm{a}$ & $6.7 \mathrm{a}$ & $40.0 \mathrm{~b}$ & $0.0 \mathrm{a}$ & $0.0 \mathrm{a}$ & $5.0 \mathrm{a}$ & $85.0 \mathrm{a}$ & $0.0 \mathrm{a}$ & $0.0 \mathrm{a}$ & $40.0 \mathrm{ab}$ & $0.0 \mathrm{a}$ & $0.0 \mathrm{a}$ & $0.0 \mathrm{a}$ & $90.0 \mathrm{a}$ \\
\hline & 40 & $5.0 \mathrm{a}$ & $25.0 \mathrm{a}$ & $55.0 \mathrm{a}$ & $0.0 \mathrm{a}$ & $5.0 \mathrm{a}$ & $0.0 \mathrm{a}$ & $85.0 \mathrm{a}$ & $5.0 \mathrm{a}$ & $5.0 \mathrm{a}$ & $55.0 \mathrm{a}$ & $0.0 \mathrm{a}$ & $10.0 \mathrm{a}$ & $0.0 \mathrm{a}$ & $85.0 \mathrm{ab}$ \\
\hline \multirow[t]{3}{*}{12} & 10 & $0.0 \mathrm{~b}$ & $14.3 \mathrm{a}$ & $12.5 \mathrm{~b}$ & $0.0 \mathrm{a}$ & $10.0 \mathrm{a}$ & $7.5 \mathrm{a}$ & $65.0 \mathrm{a}$ & $0.0 \mathrm{~b}$ & $15.0 \mathrm{a}$ & $13.3 \mathrm{~b}$ & $0.0 \mathrm{a}$ & $0.0 \mathrm{a}$ & $0.0 \mathrm{~b}$ & $65.0 \mathrm{c}$ \\
\hline & 20 & $0.0 \mathrm{~b}$ & $7.7 \mathrm{a}$ & $13.3 b$ & $0.0 \mathrm{a}$ & $10.0 \mathrm{a}$ & $5.6 \mathrm{a}$ & $80.0 \mathrm{a}$ & $0.0 \mathrm{~b}$ & $5.9 \mathrm{a}$ & $26.7 \mathrm{ab}$ & $0.0 \mathrm{a}$ & $0.0 \mathrm{a}$ & $0.0 \mathrm{a}$ & $80.0 \mathrm{~b}$ \\
\hline & 40 & $30.8 \mathrm{a}$ & $42.9 \mathrm{a}$ & $50.0 \mathrm{a}$ & $0.0 \mathrm{a}$ & $15.0 \mathrm{a}$ & $5.9 \mathrm{a}$ & $90.0 \mathrm{a}$ & $5.0 \mathrm{~b}$ & $30.0 \mathrm{a}$ & $56.3 \mathrm{a}$ & $0.0 \mathrm{a}$ & $0.0 \mathrm{a}$ & $0.0 \mathrm{a}$ & $90.0 \mathrm{a}$ \\
\hline \multirow[t]{3}{*}{14} & 10 & $0.0 \mathrm{~b}$ & $5.0 \mathrm{a}$ & $6.7 \mathrm{a}$ & $0.0 \mathrm{a}$ & $5.0 \mathrm{a}$ & $5.0 \mathrm{a}$ & $35.0 \mathrm{~b}$ & $0.0 \mathrm{a}$ & $7.1 \mathrm{a}$ & $7.0 \mathrm{a}$ & $0.0 \mathrm{a}$ & $5.0 \mathrm{a}$ & $5.0 \mathrm{a}$ & $40.0 \mathrm{~b}$ \\
\hline & 20 & $0.0 \mathrm{~b}$ & $5.6 \mathrm{a}$ & 7.1a & $0.0 \mathrm{a}$ & $0.0 \mathrm{a}$ & $0.0 \mathrm{a}$ & $50.0 \mathrm{a}$ & $0.0 \mathrm{a}$ & $9.1 \mathrm{a}$ & $14.0 \mathrm{a}$ & $0.0 \mathrm{a}$ & $0.0 \mathrm{a}$ & $0.0 \mathrm{a}$ & $45.0 \mathrm{~b}$ \\
\hline & 40 & $25.0 \mathrm{a}$ & $10.0 \mathrm{a}$ & $15.4 \mathrm{a}$ & $0.0 \mathrm{a}$ & $10.0 \mathrm{a}$ & $0.0 \mathrm{a}$ & $47.0 \mathrm{a}$ & $11.1 \mathrm{~b}$ & $12.5 \mathrm{a}$ & $19.0 \mathrm{a}$ & $0.0 \mathrm{a}$ & $7.5 \mathrm{a}$ & $0.0 \mathrm{a}$ & $50.0 \mathrm{a}$ \\
\hline
\end{tabular}

Table 3 shows the percentage reduction of fungal growth in Egusi kernels treated with different concentrations of $X$. aethiopica. A. tamarii and $A$. niger had maximum growth reduction at $40 \mathrm{~g} \mathrm{~kg}^{-1}$ treatment while $A$. flavus recorded the highest growth reduction of $50 \%$ at 20 and $40 \mathrm{~g} \mathrm{~kg}^{-1}$ treatment. For F. solani, $20 \mathrm{~g} \mathrm{~kg}^{-1}$ treatment gave the highest growth reduction of $75.0 \%$, followed by $10 \mathrm{~g} \mathrm{~kg}^{-1}$ treatment (70\% reduction) while $40 \mathrm{~g} \mathrm{~kg}^{-1}$ had the least growth reduction (30\%) after 2 weeks of treatment. Percentage growth reduction gradually declined as the storage period increased. $X$. aethiopica at various concentrations only had effect on Rhizopus sp. growth after 2 weeks of treatment. The growth of $P$. aurantiogriseum was effectively inhibited up to $14^{\text {th }}$ week after storage at all treatment levels because up to $20 \%$ growth reduction was recorded at $10 \mathrm{~g} \mathrm{~kg}^{-1}$ treatment even at the $14^{\text {th }}$ week. $P$. citrinum growth was also effectively reduced by more than $20 \%$ by the various levels of the botanical treatments throughout the 14 weeks of Egusi storage (Table 3). The second experiment gave similar results. $A$. tamarii had maximum growth reduction at $40 \mathrm{~g} \mathrm{~kg}^{-1}$ treatment while A. flavus growth reduction of $66.7 \%$ was recorded at $20 \mathrm{~g} \mathrm{~kg}^{-1}$; although not significantly different from $40 \mathrm{~g} \mathrm{~kg}^{-1}$ treatment which had $55.6 \%$ growth reduction at two weeks after storage. However, at $4^{\text {th }}$ week after storage $40 \mathrm{~g} \mathrm{~kg}^{-1}$ treatment recorded a growth reduction of $61.1 \%$ while $20 \mathrm{~g} \mathrm{~kg}^{-1}$ had $66.7 \%$ growth reduction. Percentage growth reduction declined gradually as the storage period increased. After one month of treatment $O$. gratissimum was not effective in inhibiting the growth of Rhizopus sp. The growth of $P$. aurantiogriseum was effectively inhibited up to $8^{\text {th }}$ week, $10^{\text {th }}$ week and $12^{\text {th }}$ week after storage at 10,20 and $40 \mathrm{~g} \mathrm{~kg}^{-1}$ treatment, respectively. Growth of $P$. citrinum was effectively reduced by up to $20 \%$ by the various levels of the botanical treatments throughout the 14 weeks of Egusi storage. The second experiment gave similar results. Forty (40) $\mathrm{g} \mathrm{kg}^{-1}$ treatment generally had the highest growth reduction for the various fungal isolates except for A. flavus, where $20 \mathrm{~g} \mathrm{~kg}^{-1}$ treatment gave the highest growth reduction of $80 \%$. This was followed by $40 \mathrm{~g} \mathrm{~kg}^{-1}$ treatment which had $65.0 \%$ growth reduction $P$. citrinum growth was still effectively reduced by up to $20.0 \%$ and above by the various levels of the botanical treatments throughout the 14 weeks of Egusi storage except at 2.0\% treatment in the $14^{\text {th }}$ week after storage where only $10.0 \%$ reduction was recorded (Table 4 ). 
Table 3: Effect of different concentrations of X. aethiopica on fungal contaminants of stored Egusi kernels at different weeks after storage

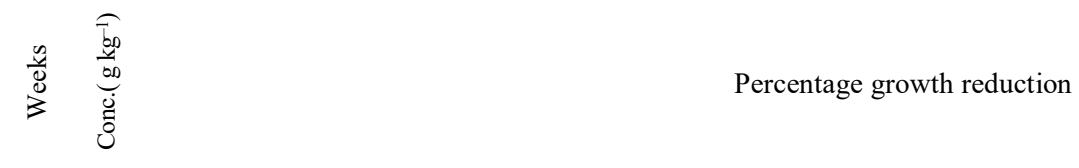

\begin{tabular}{|c|c|c|c|c|c|c|c|c|c|c|c|c|c|c|c|}
\hline \multirow[b]{3}{*}{2} & \multirow[b]{3}{*}{10} & \multicolumn{5}{|c|}{ First experiment (2012) } & \multirow{3}{*}{ TAM } & \multirow[b]{2}{*}{ YEL } & \multirow[b]{2}{*}{ FLV } & \multirow[b]{2}{*}{ NIG } & \multicolumn{3}{|c|}{ Second experiment (2013) } & \multirow[b]{2}{*}{ TAM } & \multirow[b]{2}{*}{ YEL } \\
\hline & & FLV & NIG & PUR & RHZ & SOL & & & & & PUR & RHZ & SOL & & \\
\hline & & $16.7 \mathrm{~b}$ & $35.0 \mathrm{~b}$ & $100.0 \mathrm{a}$ & $20.0 \mathrm{c}$ & $70.0 \mathrm{a}$ & & $100.0 \mathrm{a}$ & $5.6 \mathrm{~b}$ & $25.0 \mathrm{~b}$ & $100.0 \mathrm{a}$ & $20.0 \mathrm{c}$ & $55.0 \mathrm{~b}$ & $20.0 \mathrm{c}$ & $100.0 \mathrm{a}$ \\
\hline & 20 & $50.0 \mathrm{a}$ & $50.0 \mathrm{ab}$ & $100.0 \mathrm{a}$ & $30.0 \mathrm{~b}$ & $75.0 \mathrm{a}$ & $45.0 \mathrm{a}$ & $100.0 \mathrm{a}$ & $66.7 \mathrm{a}$ & $65.0 \mathrm{a}$ & $100.0 \mathrm{a}$ & $30.0 \mathrm{~b}$ & $70.0 \mathrm{ab}$ & $40.0 \mathrm{~b}$ & $100.0 \mathrm{a}$ \\
\hline \multirow{3}{*}{4} & 40 & $50.0 \mathrm{a}$ & $65.0 \mathrm{a}$ & $100.0 \mathrm{a}$ & $40.0 \mathrm{a}$ & $30.0 \mathrm{~b}$ & $55.0 \mathrm{a}$ & $100.0 \mathrm{a}$ & $55.6 \mathrm{a}$ & $55.0 \mathrm{a}$ & $100.0 \mathrm{a}$ & $40.0 \mathrm{a}$ & $25.0 \mathrm{~b}$ & $60.0 \mathrm{a}$ & $100.0 \mathrm{a}$ \\
\hline & 10 & $22.2 \mathrm{~b}$ & $25.0 \mathrm{~b}$ & $85.0 \mathrm{a}$ & $0.0 \mathrm{~b}$ & $30.0 \mathrm{~b}$ & $15.0 \mathrm{~b}$ & $90.0 \mathrm{~b}$ & $22.2 \mathrm{~b}$ & $25.0 \mathrm{~b}$ & $95.0 \mathrm{~b}$ & $5.0 \mathrm{a}$ & $35.0 \mathrm{~b}$ & $20.0 \mathrm{a}$ & $100.0 \mathrm{a}$ \\
\hline & 20 & $50.0 \mathrm{a}$ & $31.3 \mathrm{ab}$ & $97.5 \mathrm{a}$ & $10.0 \mathrm{~b}$ & $70.0 \mathrm{a}$ & $30.0 \mathrm{~b}$ & $100.0 \mathrm{a}$ & $66.7 \mathrm{a}$ & $56.3 \mathrm{ab}$ & $100.0 \mathrm{a}$ & $5.0 \mathrm{a}$ & $75.0 \mathrm{ab}$ & $40.0 \mathrm{a}$ & $100.0 \mathrm{a}$ \\
\hline \multirow{4}{*}{6} & 40 & $38.9 \mathrm{a}$ & $65.0 \mathrm{a}$ & $100.0 \mathrm{a}$ & $25.0 \mathrm{a}$ & $25.0 \mathrm{~b}$ & $65.0 \mathrm{a}$ & $100.0 \mathrm{a}$ & $61.1 \mathrm{a}$ & $66.7 \mathrm{a}$ & $100.0 \mathrm{a}$ & $15.0 \mathrm{a}$ & $15.0 \mathrm{~b}$ & $65.0 \mathrm{a}$ & $100.0 \mathrm{a}$ \\
\hline & 10 & $5.6 \mathrm{a}$ & $20.0 \mathrm{~b}$ & $43.0 \mathrm{~b}$ & $0.0 \mathrm{a}$ & $20.0 \mathrm{ab}$ & $15.0 \mathrm{a}$ & $80.0 \mathrm{~b}$ & $5.6 \mathrm{a}$ & $25.0 \mathrm{a}$ & $65.0 \mathrm{~b}$ & $0.0 \mathrm{a}$ & & $10.0 \mathrm{a}$ & $80.0 \mathrm{~b}$ \\
\hline & 20 & $10.0 \mathrm{a}$ & $35.0 \mathrm{~b}$ & $60.0 \mathrm{~b}$ & $0.0 \mathrm{a}$ & $15.0 \mathrm{~b}$ & $10.0 \mathrm{a}$ & $90.0 \mathrm{a}$ & $10.0 \mathrm{a}$ & $30.0 \mathrm{a}$ & $60.0 \mathrm{~b}$ & $0.0 \mathrm{a}$ & $20.0 \mathrm{a}$ & $15.0 \mathrm{a}$ & $90.0 \mathrm{a}$ \\
\hline & 40 & $16.7 \mathrm{a}$ & $66.7 \mathrm{a}$ & $100.0 \mathrm{a}$ & $0.0 \mathrm{a}$ & $30.0 \mathrm{a}$ & $20.0 \mathrm{a}$ & $92.5 \mathrm{a}$ & $16.7 \mathrm{a}$ & $44.4 \mathrm{a}$ & $100.0 \mathrm{a}$ & $0.0 \mathrm{a}$ & $20.0 \mathrm{a}$ & $15.0 \mathrm{a}$ & $87.5 \mathrm{a}$ \\
\hline \multirow{3}{*}{8} & 10 & $5.6 \mathrm{a}$ & $25.0 \mathrm{~b}$ & $35.0 \mathrm{~b}$ & $0.0 \mathrm{a}$ & $5.0 \mathrm{~b}$ & $15.0 \mathrm{a}$ & $60.0 \mathrm{c}$ & $11.1 \mathrm{a}$ & $12.5 \mathrm{a}$ & $40.0 \mathrm{~b}$ & $0.0 \mathrm{a}$ & $5.0 \mathrm{a}$ & $10.0 \mathrm{a}$ & $60.0 \mathrm{c}$ \\
\hline & 20 & $10.0 \mathrm{a}$ & $15.0 \mathrm{ab}$ & $45.0 \mathrm{~b}$ & $0.0 \mathrm{a}$ & $10.0 \mathrm{~b}$ & $10.0 \mathrm{a}$ & $70.0 \mathrm{~b}$ & $11.1 \mathrm{a}$ & $30.0 \mathrm{a}$ & $55.0 \mathrm{~b}$ & $0.0 \mathrm{a}$ & $5.0 \mathrm{a}$ & $15.0 \mathrm{a}$ & $75.0 \mathrm{~b}$ \\
\hline & 40 & $15.0 \mathrm{a}$ & $50.0 \mathrm{a}$ & $100.0 \mathrm{a}$ & $0.0 \mathrm{a}$ & $35.0 \mathrm{a}$ & $15.0 \mathrm{a}$ & $90.0 \mathrm{a}$ & $20.0 \mathrm{a}$ & $40.0 \mathrm{a}$ & $100.0 \mathrm{a}$ & $0.0 \mathrm{a}$ & $25.0 \mathrm{a}$ & $10.0 \mathrm{a}$ & $90.0 \mathrm{a}$ \\
\hline \multirow[t]{3}{*}{10} & 10 & $5.0 \mathrm{a}$ & $25.0 \mathrm{a}$ & $15.0 \mathrm{bc}$ & $0.0 \mathrm{a}$ & 5. & 10. & 40 & 5. & $12.5 \mathrm{a}$ & & $0.0 \mathrm{a}$ & & $5.0 \mathrm{a}$ & $70.0 \mathrm{~b}$ \\
\hline & 20 & $15.0 \mathrm{a}$ & $18.8 \mathrm{a}$ & $25.0 \mathrm{~b}$ & $0.0 \mathrm{a}$ & $10.0 \mathrm{~b}$ & $10.0 \mathrm{a}$ & 82.5 & $15.0 \mathrm{a}$ & $18.8 \mathrm{a}$ & $25.0 \mathrm{~b}$ & $0.0 \mathrm{a}$ & $10.0 \mathrm{a}$ & $7.5 \mathrm{a}$ & $80.0 \mathrm{aa}$ \\
\hline & 40 & $15.0 \mathrm{a}$ & $20.5 \mathrm{a}$ & $100.0 \mathrm{a}$ & $0.0 \mathrm{aa}$ & $30.0 \mathrm{a}$ & $5.0 \mathrm{a}$ & $80.0 \mathrm{a}$ & $15.0 \mathrm{a}$ & $30.0 \mathrm{a}$ & $100.0 \mathrm{a}$ & $0.0 \mathrm{a}$ & $10.0 \mathrm{a}$ & $10.0 \mathrm{a}$ & $80.0 \mathrm{a}$ \\
\hline \multirow{3}{*}{12} & 10 & $6.7 \mathrm{a}$ & $20.0 \mathrm{a}$ & $20.0 \mathrm{c}$ & $0.0 \mathrm{a}$ & $8.3 \mathrm{~b}$ & $7.5 \mathrm{a}$ & $25.0 \mathrm{c}$ & 7.1 ab & $10.5 \mathrm{~b}$ & $20.0 \mathrm{c}$ & $0.0 \mathrm{a}$ & $0.0 \mathrm{~b}$ & $5.0 \mathrm{~b}$ & $40.0 \mathrm{~b}$ \\
\hline & 20 & $21.4 \mathrm{a}$ & $21.1 \mathrm{a}$ & $23.1 \mathrm{~b}$ & $0.0 \mathrm{a}$ & $10.0 \mathrm{~b}$ & $15.0 \mathrm{a}$ & $60.0 \mathrm{~b}$ & $21.4 \mathrm{ab}$ & $12.5 \mathrm{ab}$ & $23.1 \mathrm{~b}$ & $0.0 \mathrm{a}$ & $10.0 \mathrm{a}$ & $20.0 \mathrm{ab}$ & $50.0 \mathrm{a}$ \\
\hline & 40 & $10.0 \mathrm{a}$ & $16.7 \mathrm{~b}$ & $100.0 \mathrm{a}$ & $0.0 \mathrm{a}$ & $30.0 \mathrm{a}$ & $6.3 \mathrm{a}$ & $68.8 \mathrm{a}$ & $20.0 \mathrm{a}$ & $16.7 \mathrm{a}$ & $100.0 \mathrm{a}$ & $0.0 \mathrm{a}$ & $20.0 \mathrm{a}$ & $7.1 \mathrm{~b}$ & $75.0 \mathrm{a}$ \\
\hline \multirow[t]{3}{*}{14} & 10 & $4.4 \mathrm{a}$ & $10.0 \mathrm{a}$ & $10.0 \mathrm{~b}$ & $0.0 \mathrm{a}$ & $6.3 \mathrm{~b}$ & $5.3 \mathrm{a}$ & $18.8 \mathrm{~b}$ & $0.0 \mathrm{a}$ & $0.0 \mathrm{a}$ & $8.3 \mathrm{c}$ & $0.0 \mathrm{a}$ & $0.0 \mathrm{c}$ & $5.0 \mathrm{~b}$ & $35.0 \mathrm{~b}$ \\
\hline & 20 & $0.0 \mathrm{a}$ & $6.3 \mathrm{ab}$ & $20.0 \mathrm{~b}$ & $0.0 \mathrm{a}$ & 7.7ab & $15.0 \mathrm{a}$ & $56.3 \mathrm{~b}$ & $0.0 \mathrm{a}$ & $5.6 \mathrm{a}$ & $12.5 \mathrm{~b}$ & $0.0 \mathrm{a}$ & $7.7 \mathrm{~b}$ & $10.0 \mathrm{ab}$ & $50.0 \mathrm{ab}$ \\
\hline & 40 & $4.4 \mathrm{a}$ & $7.1 \mathrm{~b}$ & $85.0 \mathrm{a}$ & $0.0 \mathrm{a}$ & $21.4 \mathrm{a}$ & $5.9 \mathrm{a}$ & $68.8 \mathrm{a}$ & $10.0 \mathrm{a}$ & $10.0 \mathrm{a}$ & $90.0 \mathrm{a}$ & $0.0 \mathrm{a}$ & $11.1 \mathrm{a}$ & $5.9 \mathrm{~b}$ & $62.5 \mathrm{a}$ \\
\hline
\end{tabular}

Table 4: Effect of different concentrations of $O$. gratissimum on fungal contaminants of stored Egusi kernels at different weeks after storage

\begin{tabular}{|c|c|c|c|c|c|c|c|c|c|c|c|c|c|c|c|}
\hline \multirow{3}{*}{$\frac{\tilde{v}}{\tilde{d}}$} & 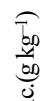 & \multicolumn{14}{|c|}{ Percentage growth reduction } \\
\hline & & & & First ex & eriment & 2012) & & & & & Second & xperime & t(2013) & & \\
\hline & & FLV & NIG & PUR & RHZ & SOL & TAM & YEL & FLV & NIG & PUR & RHZ & SOL & TAM & YEL \\
\hline \multirow[t]{3}{*}{2} & 10 & $25.0 \mathrm{~b}$ & $30.0 \mathrm{a}$ & $75.0 \mathrm{~b}$ & $20.0 \mathrm{c}$ & $50.0 \mathrm{~b}$ & $10.0 \mathrm{~b}$ & $100.0 \mathrm{a}$ & $35.0 \mathrm{~b}$ & $25.0 \mathrm{a}$ & $70.0 \mathrm{~b}$ & $20.0 \mathrm{~b}$ & $60.0 \mathrm{a}$ & $20.0 \mathrm{~b}$ & $85.0 \mathrm{a}$ \\
\hline & 20 & $65.0 \mathrm{a}$ & $40.0 \mathrm{a}$ & $100.0 \mathrm{a}$ & $40.0 \mathrm{~b}$ & $65.0 \mathrm{a}$ & $50.0 \mathrm{a}$ & $100.0 \mathrm{a}$ & $80.0 \mathrm{a}$ & $35.0 \mathrm{a}$ & $100.0 \mathrm{a}$ & $40.0 \mathrm{a}$ & $60.0 \mathrm{a}$ & $65.0 \mathrm{a}$ & $100.0 \mathrm{a}$ \\
\hline & 40 & $60.0 \mathrm{a}$ & $50.0 \mathrm{a}$ & $100.0 \mathrm{a}$ & $70.0 \mathrm{a}$ & $75.0 \mathrm{a}$ & $50.0 \mathrm{a}$ & $100.0 \mathrm{a}$ & $65.0 \mathrm{a}$ & $40.0 \mathrm{a}$ & $100.0 \mathrm{a}$ & $70.0 \mathrm{a}$ & $75.0 \mathrm{a}$ & $65.0 \mathrm{a}$ & $100.0 \mathrm{a}$ \\
\hline \multirow[t]{3}{*}{4} & 10 & $45.0 \mathrm{~b}$ & $35.0 \mathrm{~b}$ & $75.0 \mathrm{~b}$ & $0.0 \mathrm{~b}$ & $40.0 \mathrm{~b}$ & $30.0 \mathrm{a}$ & $80.0 \mathrm{~b}$ & $60.0 \mathrm{a}$ & $55.0 \mathrm{a}$ & $80.0 \mathrm{~b}$ & $5.0 \mathrm{a}$ & $25.0 \mathrm{~b}$ & $50.0 \mathrm{~b}$ & $75.0 \mathrm{~b}$ \\
\hline & 20 & $65.0 \mathrm{a}$ & $35.0 \mathrm{~b}$ & $95.0 \mathrm{a}$ & $25.0 \mathrm{a}$ & $50.0 \mathrm{a}$ & $30.0 \mathrm{a}$ & $92.5 \mathrm{a}$ & $70.0 \mathrm{a}$ & $65.0 \mathrm{a}$ & $100.0 \mathrm{a}$ & $5.0 \mathrm{a}$ & $60.0 \mathrm{~b}$ & $50.0 \mathrm{~b}$ & $95.0 \mathrm{~b}$ \\
\hline & 40 & $70.0 \mathrm{a}$ & $65.0 \mathrm{a}$ & $95.0 \mathrm{a}$ & $55.0 \mathrm{a}$ & $65.0 \mathrm{a}$ & $50.0 \mathrm{a}$ & $100.0 \mathrm{a}$ & $65.0 \mathrm{a}$ & $65.0 \mathrm{a}$ & $100.0 \mathrm{a}$ & $5.0 \mathrm{a}$ & $75.0 \mathrm{a}$ & $65.0 \mathrm{a}$ & $100.0 \mathrm{a}$ \\
\hline \multirow[t]{3}{*}{6} & 10 & $15.0 \mathrm{a}$ & $25.0 \mathrm{a}$ & $45.0 \mathrm{a}$ & $0.0 \mathrm{a}$ & $10.0 \mathrm{a}$ & $15.0 \mathrm{~b}$ & $70.0 \mathrm{~b}$ & $10.0 \mathrm{a}$ & $25.0 \mathrm{a}$ & $40.0 \mathrm{c}$ & $0.0 \mathrm{a}$ & $15.0 \mathrm{a}$ & $20.0 \mathrm{a}$ & $70.0 \mathrm{~b}$ \\
\hline & 20 & $15.0 \mathrm{a}$ & $40.0 \mathrm{a}$ & $45.0 \mathrm{a}$ & $0.0 \mathrm{a}$ & $25.0 \mathrm{a}$ & $10.0 \mathrm{a}$ & $70.0 \mathrm{~b}$ & $20.0 \mathrm{a}$ & $35.0 \mathrm{a}$ & $50.0 \mathrm{~b}$ & $0.0 \mathrm{a}$ & $25.0 \mathrm{a}$ & $10.0 \mathrm{~b}$ & $70.0 \mathrm{~b}$ \\
\hline & 40 & $40.0 \mathrm{a}$ & $55.0 \mathrm{a}$ & $60.0 \mathrm{a}$ & $0.0 \mathrm{a}$ & $20.0 \mathrm{a}$ & $10.0 \mathrm{a}$ & $90.0 \mathrm{a}$ & $35.0 \mathrm{a}$ & $40.0 \mathrm{a}$ & $60.0 \mathrm{a}$ & $0.0 \mathrm{a}$ & $20.0 \mathrm{a}$ & $10.0 \mathrm{a}$ & $92.5 \mathrm{a}$ \\
\hline \multirow[t]{3}{*}{8} & 10 & $10.0 \mathrm{~b}$ & $25.0 \mathrm{~b}$ & $35.0 \mathrm{a}$ & $0.0 \mathrm{a}$ & $10.0 \mathrm{~b}$ & $5.6 \mathrm{a}$ & $60.0 \mathrm{~b}$ & $5.0 \mathrm{a}$ & $30.0 \mathrm{a}$ & $40.0 \mathrm{a}$ & $0.0 \mathrm{a}$ & $10.0 \mathrm{a}$ & $10.0 \mathrm{a}$ & $65.0 \mathrm{a}$ \\
\hline & 20 & $15.0 \mathrm{ab}$ & $40.0 \mathrm{ab}$ & $40.0 \mathrm{a}$ & $0.0 \mathrm{a}$ & $15.0 \mathrm{ab}$ & $15.0 \mathrm{a}$ & $70.0 \mathrm{~b}$ & $10.0 \mathrm{a}$ & $45.0 \mathrm{a}$ & $30.0 \mathrm{a}$ & $0.0 \mathrm{a}$ & $25.0 \mathrm{a}$ & $15.0 \mathrm{a}$ & $75.0 \mathrm{a}$ \\
\hline & 40 & $20.0 \mathrm{a}$ & $45.0 \mathrm{a}$ & $60.0 \mathrm{a}$ & $0.0 \mathrm{a}$ & $20.0 \mathrm{a}$ & $11.1 \mathrm{a}$ & $85.0 \mathrm{a}$ & $30.0 \mathrm{a}$ & $35.0 \mathrm{a}$ & $60.0 \mathrm{a}$ & $0.0 \mathrm{a}$ & $5.0 \mathrm{a}$ & $5.6 \mathrm{a}$ & $80.0 \mathrm{a}$ \\
\hline \multirow[t]{3}{*}{10} & 10 & $5.0 \mathrm{a}$ & $20.0 \mathrm{a}$ & $25.0 \mathrm{c}$ & $0.0 \mathrm{a}$ & $10.0 \mathrm{a}$ & $15.0 \mathrm{a}$ & $70.0 \mathrm{~b}$ & $10.0 \mathrm{a}$ & $20.0 \mathrm{a}$ & $30.0 \mathrm{c}$ & $0.0 \mathrm{a}$ & $10.0 \mathrm{a}$ & $10.0 \mathrm{a}$ & $70.0 \mathrm{~b}$ \\
\hline & 20 & $11.8 \mathrm{a}$ & $20.0 \mathrm{a}$ & $55.0 \mathrm{~b}$ & $0.0 \mathrm{a}$ & $10.0 \mathrm{a}$ & $5.0 \mathrm{a}$ & $66.7 \mathrm{a}$ & $10.0 \mathrm{a}$ & $30.0 \mathrm{a}$ & $65.0 \mathrm{~b}$ & $0.0 \mathrm{a}$ & $15.0 \mathrm{a}$ & $0.0 \mathrm{~b}$ & $66.7 b$ \\
\hline & 40 & $16.7 \mathrm{a}$ & $25.0 \mathrm{a}$ & $90.0 \mathrm{a}$ & $0.0 \mathrm{a}$ & $12.5 \mathrm{a}$ & $5.0 \mathrm{a}$ & $92.5 \mathrm{a}$ & $20.0 \mathrm{a}$ & $30.0 \mathrm{a}$ & $100.0 \mathrm{a}$ & $0.0 \mathrm{a}$ & $6.3 \mathrm{a}$ & $5.6 \mathrm{~b}$ & $90.0 \mathrm{a}$ \\
\hline \multirow[t]{3}{*}{12} & 10 & $10.0 \mathrm{a}$ & $25.0 \mathrm{a}$ & $16.7 \mathrm{~b}$ & $0.0 \mathrm{a}$ & $6.7 \mathrm{a}$ & $0.0 \mathrm{a}$ & $60.0 \mathrm{~b}$ & $11.1 \mathrm{a}$ & $20.0 \mathrm{a}$ & $25.0 \mathrm{~b}$ & $0.0 \mathrm{a}$ & $20.0 \mathrm{a}$ & $20.0 \mathrm{a}$ & $60.0 \mathrm{~b}$ \\
\hline & 20 & $12.5 \mathrm{a}$ & $18.8 \mathrm{a}$ & $25.0 \mathrm{ab}$ & $0.0 \mathrm{a}$ & $8.3 a$ & $0.0 \mathrm{a}$ & $40 . \mathrm{b}$ & $14.3 \mathrm{a}$ & $6.3 a$ & $12.5 b$ & $0.0 \mathrm{a}$ & $12.5 \mathrm{a}$ & $0.0 \mathrm{a}$ & $50.0 \mathrm{~b}$ \\
\hline & 40 & $12.5 \mathrm{a}$ & $18.8 \mathrm{a}$ & $66.7 \mathrm{a}$ & $0.0 \mathrm{a}$ & $10.0 \mathrm{a}$ & $6.7 \mathrm{a}$ & $82.5 \mathrm{a}$ & $16.7 \mathrm{a}$ & $25.0 \mathrm{a}$ & $66.7 \mathrm{a}$ & $0.0 \mathrm{a}$ & $8.3 \mathrm{a}$ & $7.7 \mathrm{a}$ & $85.0 \mathrm{a}$ \\
\hline \multirow[t]{3}{*}{14} & 10 & $0.0 \mathrm{~b}$ & $11.1 \mathrm{a}$ & $9.1 \mathrm{~b}$ & $0.0 \mathrm{a}$ & $16.3 b$ & $0.0 \mathrm{a}$ & $50.0 \mathrm{ab}$ & $8.3 a$ & $17.5 \mathrm{a}$ & $14.3 \mathrm{a}$ & $0.0 \mathrm{a}$ & $15.0 \mathrm{a}$ & $0.0 \mathrm{a}$ & $45.0 \mathrm{a}$ \\
\hline & 20 & $8.3 \mathrm{ab}$ & $12.5 \mathrm{a}$ & $16.7 \mathrm{~b}$ & $0.0 \mathrm{a}$ & $7.7 \mathrm{ab}$ & $0.0 \mathrm{a}$ & $20.0 \mathrm{~b}$ & $0.0 \mathrm{a}$ & $6.3 \mathrm{a}$ & $8.3 \mathrm{a}$ & $0.0 \mathrm{a}$ & $6.3 b$ & $0.0 \mathrm{a}$ & $10.0 \mathrm{a}$ \\
\hline & 40 & $12.5 \mathrm{a}$ & $11.1 \mathrm{a}$ & $36.4 \mathrm{a}$ & $0.0 \mathrm{a}$ & $10.0 \mathrm{a}$ & $6.3 \mathrm{a}$ & $54.5 \mathrm{a}$ & $12.5 \mathrm{a}$ & $16.7 \mathrm{a}$ & $36.4 \mathrm{a}$ & $0.0 \mathrm{a}$ & $7.7 b$ & $0.0 \mathrm{a}$ & $45.5 \mathrm{a}$ \\
\hline
\end{tabular}

TAM - Aspergillus tamari; YEL - Penicillium citrinum. For each week, means with same letter in each column are not significantly different.

\section{DISCUSSION}

Fungal species belonging to nine genera were isolated and identified in Egusi kernels from the six states in South-western region of Nigeria and Aspergillus species were the most predominant. High levels of Aspergillus species population have previously been reported in Nigeria in post-harvest maize (Atehnkeng et al., 2008). The Aspergillus species recorded in the present study had previously been reported in high frequencies in Egusi (Bankole, 1993) and groundnut, another Nigerian oil seed (Ogundero, 1980). 
Besides Aspergillus, species of Penicillium, Fusarium, Trichoderma, Paecilomyces, Aternaria, Cladosporium, Sclerotium and Botryodiplodia were associated with melon seeds in Nigeria (Bankole and Joda, 2004; Chiejina, 2006; Aboloma and Ogunbusola, 2012). The bio deteriorating and aflatoxigenic fungal species spores that colonized melon must have been present in the atmosphere during sun drying and storage of the seeds. The fungi could have been introduced during exposure and direct contact of the seeds in the market (Okigbo, 2003; Gbolagade et al., 2011).

This study reveals that, all the concentrations used showed antifungal activity. Thus, they can be useful in the control of the fungi associated with stored Egusi. This agrees with the findings of Kuri et al. (2011). Ogbebor and Adekunle (2005) and Ogbebor et al. (2007) reported that extracts of $A$. sativum and $O$. basilicum demonstrated good inhibitory effect on the pathogens tested. Many workers have reported antifungal activities of different plant species and stressed the importance of plants as possible sources of natural fungicides (Ogbebor and Adekunle, 2005; Ogbebor et al., 2005; Ogbebor et al., 2007; Ogbebor and Adekunle, 2008; Shovan et al., 2008; Oyewole and Abalaka, 2012).

Many reports exist on the use of botanicals against the plant pathogenic fungi. For example, $O$. basilicum and $A$. sativum on Colletotrichum gloeosporioides (Penz). Allium cepa L., against Alternaria tenuis and Curvularia lunata Wakker, $X$. against Proteus mirabilis Hauser, Candida albicans Berkh and Staphylococcus aureus (Misra and Dixit, 1976; Okigbo et al., 2005; Ogbebor et al., 2007). This shows that these botanicals contain bioactive ingredients that are inhibitory to the growth of these pathogens. The antifungal activities of botanicals were supported by many other investigators; neem oil, betel (Piper betel L.) leaf extract, Psidium guajava L. (Hema et al., 2009), Thymus vulgaris (LINN.), Zingiber officinale, Cymbopogon citrates Stapf (lemon grass) (Zeringue et al., 2001; Chalfoun et al., 2004; Neguefact et al., 2004; Faria et al., 2006; Kumar et al., 2007; Srichana et al., 2009; Bahraminejad, 2012). The presence of antinutritional factors in the botanical species is responsible for their broad antimicrobial activity. The antifungal activity of $X$. aethiopica and $O$. gratissimum may be due to the presence of antinutritional factors in them.

\section{CONCLUSION}

It is clear from the above observations that all the botanicals $(O$. gratissimum, $P$. guineense and $X$. aethiopica) investigated proved to be useful in the management of postharvest/storage fungi. Results obtained with the botanicals in this study confirmed the importance of these plant species as exhibiting antifungal properties both in the in vitro and in vivo experiments. The present investigation is an important step in preventing contamination of seeds with botanicals, which are eco-friendly for the management of the important seed borne fungi. Therefore, exploitation of naturally available chemicals from plant protection will play a prominent role in development of future commercial pesticides for crop protection strategies, with special reference to the management of plant diseases. This can also be usefully exploited in the protection of foods from mycotoxin contamination.

\section{REFERENCES}

Aberkane A., Cuenca-Estrella M., Gomez-Lopez A., Petrikkou E., Mellado E., Monzón A., RodriguezTudela J.L. and Eurofung N. (2002). Comparative evaluation of two different methods of inoculums preparation for antifungal susceptibility testing of filamentous fungi. J. antimicrob. chem., 50, 719-722

Aboloma R.I., Onifade A.K. and Adetuyi F.C. (2009). Control of fungal deterioration of some fruits of the family Cucurbitaceae using plant materials. Nig. J. Mycol., 2 (1), 22- 36

Aboloma R.I. and Ogunbusola E.M. (2012). Fungi associated with Irvingia gabonensis (ogbono) and Colocynthis citrullus (Egusi) seeds sold in markets in Ado-Ekiti, Ekiti State Nigeria. J. Microbiol. Biotech. Res., 2 (3), 423-426

Aboloma R.I., Onifade A.K. and Adetuyi F.C. (2012). Effect of deterioration on the proximate composition of some fruits of the family Cucurbitaceae. $J$. Microbiol. Biotech. Res., 2 (1), 240-243

Alexopoulos C.J., Mims C.W. and Blackwell M. (2002). Introductory Mycology (5th ed.), John Wiley and Sons, INC., p. 69.

Atehnkeng J., Ojiambo P.S., Donner M., Ikotun T., Sikora R.A., Cotty P.J. and Bandyopadhyay R. (2008). Distribution and toxigenicity of Aspergillus species isolated from maize kernels from three agroecological zones in Nigeria. Int. J. Food Microbiol., 122 (1-2), 74-84

Atehnkeng J., Ojiambo P.S., Cotty P.J. and Bandyopadhyay R. (2014). Field efficacy of a mixture of atoxigenic Aspergillus flavus Link: Fr vegetative compatible groups in preventing aflatoxin contamination in maize (Zea mays L.). Biol. Control, 72, 62-70

Ayodele O.J. and Salami A.E. (2006). Physiological response of two variants of Egusi melon (Citrullus lanatus) to plant population density in a humid environment. J. Food, Agric. and Envt., 4 (3 and 4), 110-113

Bahraminejad S. (2012). In vitro and In vivo antifungal activities of Iranian plant species against Pythium aphanidermatum. Ann. Biol. Res., 3 (5), 2134-2143

Bankole S.A. (1993). Moisture content, mould invasion and seed germinability of stored melon. Mycopathologia, 122, 123-126

Bankole S.A. and Joda A.O. (2004). Effect of lemon grass (Cymbopogon citratus) powder and essential oil on mould deterioration and aflatoxin contamination of melon seeds (Colocynthis citrullus L.). Afr. J. Biotech., 3 (1), 52-59 
Barnett H.L. and Hunter B.B. (1999) Illustrated Genera of Imperfect Fungi (4 $4^{\text {th }}$ ed.), The American Phytopathological Soc. St. Paul, Minnesota, pp. 7-201

Chalfoun S.M., Pereira M. Lúcio C.M., Resende V., Angélico C.L. and Silva R.A. (2004). Effect of powdered spice treatments on mycelial growth, sporulation and production of aflatoxins by toxigenic fungi. Ciênc. Agrotec. Lavras, 28 (4), 856-862

Chiejina N.V. (2006). Studies on seed-borne pathogens of some Nigerian melons. J. Agric., Food, Envt. and Ext., 5 (1), 13-16

Dusanee T. (2011). Control of aflatoxins in agricultural products using plant extracts. KMITL Sci.and Tech. J., 11 (1), 35-42

Faria T.J., Ferreir R.S., Yassumoto L., de Souza J.R.P., Ishikawa N.K. and Barbosa A.M. (2006). Antifungal activity of essential oil isolated from Ocimum gratissimum L. (eugenol chemotype) against phytopathogenic fungi. Braz. Arch. Biol. Technol., 4, 867-871

Gbolagade J., Ajayi I. and Omitade Y. (2011). Nutritional compositions, fungi and aflatoxins detection in stored 'gbodo' fermented (Dioscorea rotundata) and 'elubo ogede' fermented (Musa parasidiaca) from south western Nigeria. Afr. J. Food Sci., 5 (2), 105-110

Giridher P. and Ready S.M.P. (1997). Incidences of mycotoxin producers on spices from Andhra Pradesh. J. Indian Bot. Soc., 76, 161-164

Hema R., Kumaravel S. and Elanchezhiyan N. (2009). Antimicrobial activity of some of the South-Indian spices and herbals against food pathogens. Global $J$. Pharmacol., 3, 38-40

Kumar R., Mishra A.K, Dubey N.K. and Tripathi Y.B. (2007). Evaluation of Chenopodium ambrosioides oil as a potential source of antifungal, anti-aflatoxigenic and antioxidant activity. Int. J. Food Microbiol., 115, 159-164

Kuri S.K., Islam R.M. and Mondal U. (2011). Antifungal potentiality of some botanical extracts against important seed borne fungal pathogen associated with brinjal seeds (Solanum melongena L.). J. Agric. Technol., 7 (4), 1139-1153

Misra S.B. and Dixit S.N. (1976). Fungicidal spectrum of the leaf extract of Allium sativum. Indian Phytopathol, 29, 448-449

Neguefact J., Leth V., AmvamZollo P.H. and Mathur S.B. (2004). Evaluation of five essential oils from aromatic plants of Cameroon for controlling food spoilage and mycotoxin producing fungi. Int. J. Food Microbiol., 94, 329-334

Ogbebor O.N. and Adekunle A.T. (2005). Inhibition of conidial germination of mycelial growth of Corynespora cassiicola (Berk and Curt) of rubber (Hevea brasiliensis Muell. Arg.) using extracts of some plants. Afr. J. Biotech., 4 (9), $996-1000$

Ogbebor O.N., Adekunle A.T. and Enobakhare DA. (2005). Inhibition of Colletotrichum gloeosporioides (Penz) Sae. causal organism of rubber (Hevea brasiliensis Muell. Arg.) leaf spot using plant extracts. Afr. J. Biotech., 6 (3), 213-218
Ogbebor O.N. and Adekunle A.T. (2008). Inhibition of Drechslera heveae (Petch) M.B. Ellis, causal organism of bird's eye spot disease of rubber (Hevea brasiliensis Muell Arg.) using plant extracts. Afr. J. Biotech., 4 (1), 19-26

Ogbebor N.O., Adekunle A.T. and Enobakhare D.A. (2007). Inhibition of Colletotrichum gloeosporioides (Penz) Sac. Causal organism of rubber (Hevea brasiliensis Muell. Agr.) leaf spot using plant extracts. Afr. J. Biotech., 6, 213-218

Ogundero V.W. (1980). Lipase activities of thermophilic fungi from mouldy groundnuts in Nigeria. Mycol., 72, $118-26$

Okigbo R.N. (2003). Fungi associated with peels of postharvest yams in storage. Global Journal of Pure and Applied Sciences, 9 (1), 19-23

Okigbo R.N., Mbajiuka C.S. and Njoku C.O. (2005). Antimicrobial potentials of Xylopia aethopica (uda) and Ocimum gratissimum L. on some pathogens of man. Int. J. Mol. Med., 1 (4), 392-397

Oyewole O.A. and Abalaka M.E. (2012). Antimicrobial activities of Telfairia occidentalis (fluted pumpkins) leaf extract against selected intestinal pathogens. $J$. Health Sci., 2 (2), 1-4

Samson R.A., Hoekstra E.S. and Frisvad J.C. (2004). Introduction to food-borne fungi ( $7^{\text {th }}$ ed.), Central Bureauvoor Schimmel cultures, pp 300-389

Shetty H.S. (1992). Different types of damages in seeds caused by seed-borne fungi. In: S.B. Mathur and J. Jorgensen (eds.), Seed pathology (pp. 53-62). Proc. of Technical Center for Agricultural and Rural Cooperation Seminar held in Copenhagen Denmark. Technical Center for Agricultural and Rural Cooperation, Wageningen, Netherlands, pp. 412

Shovan L.R., Bhuiyan M.K.A., Begum J.A. and Pervez Z. (2008). In vitro control of Colletotrichum dematium causing anthracnose of soybean by fungicides, plant extracts and Trichoderma harzianum. Int. J. Sustain. Crop Prod., 3 (3), 10-17

Srichana D., Phumruan A. and Chongkid B. (2009). Inhibition effects of betel leaf extract on the growth of Aspergillus flavus and Fusarium verticillioides (Thammasat). Int. J. Sci. Tech., 14, 74-77

Van der Vossen H.A.M., Denton O.A. and El Tahir I.M. (2004). Citrullus lanatus (Thunb.) Matsum.and Nakai. [Internet] Record from Protabase.Grubben, G.J.H. and Denton, O.A. (Editors). PROTA (Plant Resources of Tropical Africa / Ressources végétales de l'Afrique tropicale), Wageningen, Netherlands. $737 \mathrm{pp}$.

Yallappa R., Nandagopal B. and Thimmappa S. (2012). Botanicals as grain protectants. Psyche, (2012), 13. Article ID 646740. Doi:10.1155/2012/646740

Zeringue H.J., Shih B.Y. and Bhatnagar D. (2001). Effects of clarified neem oil on growth and aflatoxin B1 formation in submerged and plate cultures of aflatoxigenic Aspergillus spp. Phytoparasitica, 29, 361-366 